\title{
Europäisches Marktrecht vs. soziales Europa
}

\author{
Antoine Lyon-Caen
}

I. Einleitung

1. Zwischen Überraschung und vorhersehbarer Entscheidung 9

2. Ausblick

II. Die Trennung von Marktrecht und Arbeitsrecht 11

1. Die Gleichstellung des Arbeitsrechts

2. Die Trennung als Bestandteil der demokratischen Verfassung der Europäischen Staaten

3. Die demokratische Verfassung der Mitgliedsstaaten als Säule des Europäischen Einigungswerkes

III. Der Beginn einer gefährlichen Beziehung 14

1. Die Tendenz in Richtung einer neuen Unterordnung 14

2. Die Gefahren der Abhängigkeit

\section{Einleitung}

\section{Zwischen Überraschung und vorhersehbarer Entscheidung}

Es ist nicht sicher, dass jeder in ihnen das Gleiche sucht oder findet. Aber eines ist sicher: die drei Urteile des Europäischen Gerichtshofes, die unter den Namen Viking, Laval und Rüffert berühmt geworden sind ${ }^{1}$, lösen auch über die Fachkreise der Juristen hinaus ein reges Interesse aus. Diese Urteile behandeln die schwierigen Beziehungen zwischen der Niederlassungs- und Dienstleistungsfreiheit einerseits und dem Arbeitsrecht andererseits.

Aus praktischen Erwägungen und insbesondere, um sich der Fachsprache zu bedienen, wird vorgeschlagen vom Marktrecht zu sprechen. Dessen Komponenten sind, neben anderen Grundfreiheiten, die Niederlassungsfreiheit und die Dienstleistungsfreiheit sowie das Wettbewerbsrecht; zusammen gefasst schaffen diese so bezeichneten Rege-

1 EuGH vom 11. Dezember 2007, Viking C-488/05, Slg. 2007, I-10779; vom 18. Dezember 2007, Laval, C-341/05, Slg. 2007, I-11767; vom 3. April 2008, Rüffert, C-346/06. 
lungen einen Markt mit dem Ziel, einen funktionierenden Wettbewerb zu gewährleisten. Das Kernstück dieser Urteile bildet die Kollision des Marktrechtes, als Teil des europäischen Einigungswerkes, mit dem Arbeitsrecht, das nationale Wurzeln hat.

Schon früher war der Gerichtshof in Luxemburg Schauplatz einer solchen Kollision. ${ }^{2}$ Er legte sie jedoch nicht offen dar. Er hat den analytischen Rahmen festgelegt und die Denkweisen aufgezeigt, um die Modalitäten für eine solche Kollision festzulegen. Mit einem Geschick, um das andere ihn beneiden würden, scheint er in den Urteilen Viking, Laval und Rüffert das vorher zum Ausdruck gebrachte, wieder aufgenommen und fortgesetzt zu haben. Kurz gesagt, die Kontinuität ist gewahrt, jede Überraschung ausgeschlossen, sofern sie nicht gespielt ist.

Dieser erste Eindruck ist jedoch nicht gerechtfertigt, denn die Kollision, die er in den jüngsten Fällen zu beurteilen hat, weist neuartige Züge auf. Was der Gerichtshof glaubt, dem europäischen Marktrecht - mit seinen Komponenten Niederlassungs- und Dienstleistungsfreiheit - gegenüberstellen zu können, ist das Rechtssystem, d.h. das nationale Rechtssystem der industriellen Beziehungen. Es wird vorgeschlagen, dieses als „Recht der industriellen Beziehungen“ zu bezeichnen. Der Begriff industrielle Beziehungen wird verwendet, um die gesamten Beziehungen zu bezeichnen, d.h. Konfrontation, Verhandlungen oder Zusammenarbeit, die sich zwischen Arbeitnehmern und ihren Organisationen sowie den Arbeitgebern und deren zuständigen Organisationen und teilweise auch mit den staatlichen Behörden entwickeln. Hinsichtlich der Regelungen zur Überwachung und Gestaltung dieser Beziehungen braucht wohl kaum daran erinnert zu werden, dass sie unterschiedlichsten Ursprungs sind: es handelt sich weder zwangsläufig um Heteronomie noch unbedingt um Autonomie, die Kombinationen sind je nach den nationalen, sektoriellen und lokalen Erfahrungen vielfältig und unterschiedlich.

Die Situation bei dieser Kollision ist ganz neu, denn was der Gerichtshof in seinen jüngsten Urteilen anspricht, ist das Rechtssystem der industriellen Beziehungen, auf das er bisher nicht offen eingegangen ist.

\section{Ausblick}

Durch die neuartigen Züge der Kollision entstehen bestimmte Anforderungen. Die erste betrifft die Art und Weise wie diese Urteile zu lesen und auszulegen sind. Es ist sicherlich erforderlich den Aufbau, den Zusammenhang und das rechtliche Regelwerk zu verstehen. Aber man muss sich auch bemühen zu verstehen, was der Gerichtshof tut,

2 S. insbesondere EuGH vom 5. Juni 1997, SETTG, C-398/95, Slg. 1997, I-3091, worin der Gerichtshof unvorsichtig erklärte, dass "die Wahrung des sozialen Friedens als Mittel, um einen Tarifkonflikt zu beenden keinen Grund im Allgemeininteresse darstelle, der eine Einschränkung eines Grundrechts rechtfertige, das durch den Vertrag garantiert sei" (in diesem Falle handelte es sich um die Dienstleistungsfreiheit). 
wenn er zum Verhältnis zwischen dem Recht der industriellen Beziehungen und dem Marktrecht Stellung bezieht.

Die hier vorgebrachte These hebt an erster Stelle hervor, was der Gerichtshof nicht für wesentlich erachtet, auf jeden Fall nicht (durch ihn) für unantastbar hält: die Trennung zwischen dem Recht der industriellen Beziehungen und dem Marktrecht. Wir haben den Eindruck, dass die Tragweite und Bedeutung dieser Urteile klarer zum Ausdruck kommen, wenn man die Bedeutung dieser Trennung unterstreicht.

\section{Die Trennung von Marktrecht und Arbeitsrecht}

\section{Die Gleichstellung des Arbeitsrechts}

In den westeuropäischen Gesellschaften hat sich das Arbeitsrecht durch die Gleichstellung gegenüber dem Marktrecht herausgebildet, das mit unterschiedlichen Begriffen bezeichnet wurde, die nicht in Vergessenheit geraten dürfen: hier Handelsfreiheit woanders freedom of trade. Es ist nicht so, als hätte es vor dieser Gleichstellung keine Regelungen im Bereich der Arbeit gegeben, aber sie fielen eher in den Zuständigkeitsbereich einer Arbeitspolizei, ein mehr oder weniger unabhängiger Teil der Polizei mit Zuständigkeiten für die Märkte. Wie hat sich diese Gleichstellung in großen Zügen abgespielt? Sie erfolgte aufgrund der institutionellen Neuerungen, die einen Bruch mit den Prinzipien und der dem Wettbewerbsmarkt eigenen Rationalität bedeuten; aus diesem Grund aber auch wegen der beispielhaften Rolle, die sie im Rahmen der Gleichstellung gespielt haben, bilden sie immer noch die historischen Säulen des Arbeitsrechts.

Zwei Neuerungen gilt es auf jeden Fall zu erwähnen. Die Erste ist die Schaffung oder Anerkennung eines Freiraums für kollektive Maßnahmen der Arbeitnehmer. Die Wege, über die diese Anerkennung erreicht wurde, sind vielfältig. Dazu zählt zweifellos auch der Rückgang von Strafverfahren im Zusammenhang mit Bündnissen und Zusammenschlüssen von Arbeitnehmern. Jedoch wurde in einigen europäischen Ländern Straffreiheit bei kollektiven Maßnahmen im Zusammenhang mit Schadensersatzklagen eingeführt. Im Allgemeinen fand an anderer Stelle etwas später, die Proklamation der Grundrechte statt, insbesondere des Streikrechts, als verfassungsmäßiges Recht mit allen Garantien, die dieses Recht begleiten; was offensichtlich der Ausdruck diese Gleichstellung ist.

Eine zweite institutionelle Neuerung muss ebenfalls angesprochen werden und zwar: die Einführung von Verfahren oder Mechanismen zur Festlegung eines üblichen Tarifs oder Lohns, der tendenziell für alle Arbeitnehmer Anwendung finden sollte. Diese grundlegende Regelung erfolgte in einigen Fällen als Fortsetzung der vorausgegangenen institutionellen Neuerung. Dies trifft dort zu, wo der Tarifvertrag der Weg zur Einführung einer üblichen Entlohnung ist. Aber die Tarifverhandlung ist ganz und gar nicht 
der alleinige Weg dorthin; insbesondere dann nicht, wenn für bestimmte Vorhaben oder gewisse Gruppen, die von jeglichen Tarifverhandlungen ausgeschlossen sind, eine übliche Entlohnung vereinbart werden muss. Aus dieser Situation heraus entstand Ende des 19. Jahrhunderts in Frankreich das Verfahren, das der Gerichtshof in der Rechtssache Rüffert kritisiert hat. Der Zwiespalt, der sich bei der Einhaltung einer üblichen Entlohnung für die Zuschlagsempfänger eines öffentlichen Auftrags ergibt, wurde damals schon erkannt und betont: es geht darum, einen fairen Wettbewerb durchzusetzen, dessen Anwendungsbereich eingegrenzt ist und bei dem vermieden wird, dass sich die Arbeits- und Entlohnungsbedingungen verschlechtern; aus rechtsgrundsätzlichen Erwägungen geht es auch darum, die Würde der Arbeitnehmer zu wahren.

Es ist nicht nötig, hier noch weiter auszuholen: die Gleichstellung gegenüber dem Marktrecht ist erfolgt, zu Gunsten des Arbeitsrechts, der Zulassung von Kollektivmaßnahmen und der Einführung einer üblichen Entlohnung - die häufig zum Mindestlohn geworden ist -, das Eine oder Andere entspricht nicht dem Prinzip der individualistischen Optimierung oder dem optimierenden Individualismus.

\section{Die Trennung als Bestandteil der demokratischen Verfassung der Europäischen}

\section{Staaten}

In den westeuropäischen Gesellschaften hat die Trennung von Arbeitsrecht und Marktrecht, die bedingt ist durch Gleichstellung des Arbeitsrechts, eine tief greifende Bedeutung. Man kann sich logischer Weise vorstellen, dass der Aufschwung des Marktrechtes, das eine besondere Rationalität aufweist, durch einen Differenzierungseffekt möglich war. Aber der eigentliche Sinn dieser Trennung soll hier erläutert werden. Sie ist tatsächlich der Grundpfeiler der demokratischen Ordnung der westeuropäischen Gesellschaften. Um dies nachzuvollziehen, muss man sich das besondere Statut der Akteure, der Maßnahmen und Tätigkeiten, die unter das Arbeitsrecht fallen genauer ansehen. Der Arbeitnehmer als Person genießt einen Schutz, der keinem anderen Vertragspartner zuteil wird. Und dieser Schutz ist Bestandteil der demokratischen Ansprüche der europäischen Staaten. In den meisten Staaten ist die gewerkschaftliche Handlungsfreiheit, das Recht auf Tarifverhandlungen durch die Verfassung fest garantiert. Und seither sind die Tarifverhandlungen und die Kollektivmaßnahmen fest im Grundrecht der Vereinigungsfreiheit verankert, so wie es in der Europäischen Konvention zum Schutz der Menschenrechte in Artikel 11 proklamiert und garantiert wird. All diese Aussagen würden an Banalität grenzen, wenn man nicht auf den daraus resultierenden Schutz für die Arbeitnehmer, für ihre Organisationen und Aktionen vor der Übermacht der Marktregelungen hinweisen würde. Anders gesagt: der verfassungsmäßige Schutz des Arbeitnehmers als Person und das fundamentale Recht auf Organisationsfreiheit und auf Kollektivmaßnahmen haben logischerweise zur Folge - oder finden ihre notwendige Fortset- 
zung dadurch -, dass die Handlungen und Aktionen der Arbeitnehmer und ihrer Organisationen gegenüber dem Einfluss der Marktregelungen abgeschirmt sind.

Diese Äußerung kann durch ihre Bestimmtheit eine skeptische Reaktion auslösen. Jedoch hat die Trennung im verfassungsmäßigen Sinne einen grundlegenden Charakter. Dadurch wird nicht vermieden, dass die Maßnahmen und Aktionen der Arbeitnehmer und ihrer Organisationen beobachtet und begrenzt werden, aber die Bedingungen und Beschränkungen erfolgen durch speziell für diesen Zweck ausgearbeitete Regelungen. Dadurch wird auch nicht verhindert, dass die Tarifverträge und Lohnvereinbarungen in unterschiedlichem Umfang die Wettbewerbsbedingungen auf dem Markt berücksichtigen. Aber die Tatsache, dass die sozialen Protagonisten das Wettbewerbsumfeld berücksichtigen, bedeutet keinesfalls, dass sie sich den Regeln des Marktes unterwerfen.

\section{Die demokratische Verfassung der Mitgliedsstaaten als Säule des Europäischen}

\section{Einigungswerkes}

Es soll hier nicht darum gehen, erneut die Beziehungen zwischen der Verfassung der Mitgliedsstaaten und der Verfassung der Union, man könnte sogar sagen - seiner politi-

schen Verfassung - zu erörtern. Es soll nur daran erinnert werden, dass die Trennung von Arbeitsrecht und Marktrecht als Bestandteil der demokratischen Verfassung der Staaten ein Teilstück des europäischen Einigungswerkes ist; davon sollte das Gericht nicht abweichen können. Im Übrigen würdigt das Gemeinschaftsrecht bezeichnenderweise im Namen seines demokratischen Anspruchs, die normative Tätigkeit der Sozialpartner - oder das Recht der industriellen Beziehungen - so wie es von den Mitgliedsstaaten gestaltet wurde. Diese Wertschätzung wird nicht nachdrücklich betont, und es bedarf einer konstruktiven Interpretation, um aus der Achtung der Grundrechte gemäß den gemeinsamen verfassungsmäßigen Traditionen (Art. 6 EU) zu schließen, dass die im Verfassungsrecht der Mitgliedsstaaten verankerte Trennung von Arbeitsrecht und Marktrecht für die Institutionen der Gemeinschaft verbindlich ist. Aber das erstinstanzliche Gericht hat den Weg aufgezeigt; es hat nicht gezögert aufgrund des demokratischen Prinzips, auf dem die Europäische Union beruht, zu folgern, dass wenn keine parlamentarische Intervention erfolgt, die Beteiligung des Volkes an der Ausarbeitung einer Rechtsakte über die Sozialpartner stattfindet.3 Vom Artikel 137 des Vertrages geht eine doppelte Botschaft aus. Eine eindeutige Aussage, wenn er die Entlohnung und insbesondere die Vereinigungsfreiheit und das Recht auf Kollektivmaßnahmen außerhalb des Aktionsradius der Gemeinschaft ansiedelt. Diese erste Aussage könnte ausreichen, um einer Institution der Gemeinschaft jegliche Befugnis abzusprechen, das Recht und die nationalen Praktiken auf diesen Gebieten zu ändern. Aber der Text hat noch einen anderen Sinn. Denn die Entlohnungen und die Art ihrer Festlegung, sowie die Regelun-

3 EuGeI vom 17. Juni 1998, CGPME, T-135/96, Slg. 1998, II-2335 Rdnr. 89. 
gen für die Organisation und für die Kollektivmaßnahmen sind die wesentlichen Bestandteile, welche entsprechend der demokratischen Verfassung der Mitgliedsstaaten, vom Marktrecht ausgenommen sind. Der Artikel 137 ist sozusagen das Markenzeichen einer Verpflichtung der Gemeinschaft, die darin besteht, die Gleichstellung des Arbeitsrechts zu respektieren. Und dennoch...

\section{Der Beginn einer gefährlichen Beziehung}

\section{Die Tendenz in Richtung einer neuen Unterordnung}

Durch die drei jüngsten Urteile zeichnet sich eine neue Beziehung zwischen Marktrecht und Arbeitsrecht ab und, was noch viel besorgniserregender ist, dies betrifft besonders die industriellen Beziehungen. Die Trennung bewegt sich in Richtung Unterordnung. Wie manifestiert sich diese Unterordnung?

Die Regelungen, die den Markt bestimmen, d. h. die Niederlassungsfreiheit und die Dienstleistungsfreiheit haben, gemäß dem Diskurs des Gerichtshofes, den Status eines Prinzips. Der Begriff entfaltet nun seine ihm inhärente dreifache Bedeutung. Das heißt seine Stärke. Es handelt sich in erster Linie um Regelungen, die mit dem umfangreichsten Geltungsbereich ausgestattet sind. Nichts oder fast nichts entgeht ihnen. Vorbei ist es nun mit der ermutigenden Vorsicht, die der Gerichtshof bei den Verbotsregelungen für Absprachen walten ließ. Er hatte nämlich beschlossen, dass die Sozialpartner diesen nicht unterliegen, da anderenfalls die Ziele der Sozialpolitik, die ihre Absprachen rechtmäßig verfolgen, gefährdet werden. ${ }^{4}$ Folgt man den Urteilen Laval, Viking und Rüffert, so trifft dieser venünftige Vorbehalt nicht auf die Niederlassungs- und Dienstleistungsfreiheit zu, die jedoch auch auf die Organisation und das Funktionieren eines Wettbewerbsmarktes ausgerichtet sind. Dieser Bereich ist nach dem Prinzip der Allgemeingültigkeit gestaltet. Hinzu kommt noch der besondere Einfluss, den diese haben. Zur zweiten Bedeutung ist zu sagen, dass der Gerichtshof keine Neuerungen einführt und seine früheren Vorschläge wieder aufgreift. Er bereichert sie jedoch, indem er diesen Prinzipien umfassende Effizienz verleiht und die Möglichkeit hinzufügt, dass sie von allen geltend gemacht werden können.

Im Gegenzug werden die nationalen Regelungen, die Tarifverhandlungen und Kollektivmaßnahmen zulassen und garantieren sowie die Regelungen, die Lohnstandards festlegen, in ihren Beziehungen zu den gemeinschaftlichen Marktregelungen zu Ausnahmen. Was nicht heißt, dass sie ihre Anwendungsbestimmung verlieren. Aber im Falle eines angeblichen Widerspruchs zu den Marktregeln ist zu ihrer Aufrechterhaltung

4 EuGH vom 21. September 1999, Albany, C67/96, Slg. 1999, I-5751; vom 21. September 2000, Van der Wonde, C-222/98, Slg. 2000, I-7111. 
der Nachweis zu erbringen, dass sie gerechtfertigt sind. Diese Nachweisforderung kann mehr oder weniger streng ausfallen; sie kann schonungslos sein oder als ein mehr oder weniger wesentlicher Bestandteil der Verhältnismäßigkeitsprüfung berücksichtigt werden. Das Wesentliche ist dennoch die neue Aufsicht, die das Markrecht gemeinschaftlichen Ursprungs über das Recht der industriellen Beziehungen ausübt.

\section{Die Gefahren der Abhängigkeit}

Um die europäische Integration beim Namen zu nennen, die im Wesentlichen durch die Grundfreiheiten erfolgt, zieht man die Idee der negativen Integration heran. Was die negative Integration auslöst, so wie sie durch die Urteile Laval, Viking und Rüffert dargestellt wurde, ist ein Prozess der Zersetzung der Fundamente des Arbeitsrechts. Und wenn man weiß, mit welcher Kraft diese Zersetzung auf anderen Gebieten stattfand, so ist vorherzusehen, dass der Zersetzungsvorgang beträchtlich sein könnte. Gleichzeitig könnte dadurch das Gemeinschaftsrecht und auch das Marktrecht an Ansehen verlieren. Sein Eindringen in die industriellen Beziehungen, die in ihrer Gestaltung und ihren Auswirkungen auf Arbeit und Entlohnung dadurch beeinträchtigt werden, lösen soziale Proteste aus. Dies umso mehr, als seine Dynamik nur eine Einbahnstrasse ist: sie trägt niemals dazu bei, das Ungleichgewicht zwischen den Unternehmen, deren Macht durch die Erweiterung des Marktes gewachsen ist, und den geschwächten Arbeitnehmen zu verringern.

Aber die neue Bevormundung, die im Namen des Marktrechtes ausgeübt werden soll, hat eine noch tiefere Bedeutung. Wenn die Trennung des Arbeitsrechts, vor allem des Rechts der industriellen Beziehungen und des Marktrechts ein Bestandteil der demokratischen Verfassung der Mitgliedsstaaten der Union ist, wenn diese demokratische Verfassung eine Säule des europäischen Einigungswerkes ist, bzw. der politischen Verfassung Europas - wie das Suggestivwort einiger Autoren lautet -, dann bedeutet das Infragestellen dieser Trennung von Arbeitsrecht und Marktrecht, die demokratischen Ansprüche Europas noch weiter zurückzuschrauben. 
